\title{
Preparation and Characterization of Mucoadhesive Nanoparticles (NPs) Containing Quercetin and Eudragit@ RS 100 for Nasal Drug Delivery
}

\author{
Shweta R. Jawanjar*, Shital A. Chandewar, Dinesh M. Biyani, Milind J. Umekar
}

Department of Pharmaceutics, Smt. Kishoritai Bhoyar College of Pharmacy, Kamptee, Maharashtra, India

DOI: $10.36347 /$ sajp.2020.v09i02.002

| Received: 02.02.2020 | Accepted: 09.02.2020 | Published: 12.02.2020

*Corresponding author: Shweta R. Jawanjar

Abstract

Original Research Article

Quercetin nanoparticles were developed in order to accomplish poor aqueous solubility of quercetin and enhancement of drug absorption rate to nasal mucosa by increasing drug retention time using single emulsion technique. For this purpose, Eudragit ${ }^{\circledR}$ RS 100 was chosen as mucoadhesive polymer and used at different drug/polymer ratios in the nanoparticles formulations. The lyophilized nanoparticles were evaluated with respect to the drug loading, entrapment efficiency, solubility analysis, particle size, mucoadhesive property, in vitro drug release, in vitro drug diffusion, ex vivo permeation study and histopathological study, Fourier transform infrared spectroscopy (FTIR) and powder X- ray diffraction study (P-XRD) showed molecular dispersion and conversion of the drug into amorphous form. Size and surface morphology of nanoparticles was analyzed by scanning electron microscopy (SEM) and found to be spherical in shape with smooth surface. The solubility studies of QCT NPs $(61.27 \pm 0.89 \mu \mathrm{g} / \mathrm{ml})$ were found to be increased than pure QCT $(2.55 \pm 0.76 \mu \mathrm{g} / \mathrm{ml})$. Nanoparticles showed adequate mucoadhesion, the mucoadhesive potential of optimized QCT NPs showed a higher percentage of mucoadhesion $(73.45 \pm 1.16 \%)$ and do not have any destructive effect on nasal mucosa. Hence, QCT NPs based on a Eudragit RS 100 may be a promising nasal delivery system improved permeation profile for longer period of time and thereby increasing the patience compliance.

Keywords: Quercetin, Eudragit ${ }^{\circledR}$ RS100, nasal drug delivery, single emulsion technique, solubility, in vitro mucoahesion.

Copyright @ 2020: This is an open-access article distributed under the terms of the Creative Commons Attribution license which permits unrestricted use, distribution, and reproduction in any medium for non-commercial use (NonCommercial, or CC-BY-NC) provided the original author and source are credited.

\section{INTRODUCTION}

Drug delivery to the brain is challenging because of the presence of the blood-brain barrier (BBB). As a noninvasive treatment, intranasal administration bypasses the $\mathrm{BBB}$ and allows direct access to the brain through olfactory and trigeminal nerve pathways, which has led to its receiving significant attention in recent years [1, 2]. It offers advantages such as brain targeting, no gastrointestinal irritation, fast onset of action, avoidance of first pass metabolism, and fewer systemic side effects [3]. However, nasal mucociliary clearance is a significant limiting factor for nasal drug delivery [4]. It severely limits the time available for drug absorption and effectively rules out sustained nasal drug administration [5].

To improve the nasal residence time of the formulation, Eudragit@ RS100, has shown great potential, by decreasing mucociliary clearance due to its bioadhesive and permeation-enhancing properties Eudragit ${ }^{\circledR}$ RS100, a co-polymer of poly (ethylacrylate, methyl-methacrylate methacrylate), contains $4.5-6.8 \%$ of quaternary ammonium groups [6]. The latter chemical groups provide positive surface charge to the polymer, by which it may interact with negatively charged drugs or cellular surface of the target tissues. This characteristic may subsequently maximize the cellular uptake of drug-polymer complex $[7,8]$.

Quercetin is a unique bioflavonoid compound commonly found in fruits and vegetables, has been reported with a potential antioxidant, free radical scavenging, anti-inflammatory, anti-ischaemic [9], anticancer [10], antiviral, antisiabetic [11] activities and as anticoagulant property [12]. In addition, neuroprotective effects with improved morphological as well as functional activity. Quercetin is found in onions, broccoli apples, berries and red wine [13, 14] Quercetin is having low aqueous solubility (ranging from 2.15 to $7.7 \mu \mathrm{g} / \mathrm{mL}$ at $\left.25^{\circ} \mathrm{C}\right)[15,16]$ poor permeability, instability in physiological medium, less absorption, short biological half- life, extensive first pass metabolism before reaching the systemic circulation, resulting in low oral bioavailability and this have 
limited the wide application of quercetin in pharmaceutical field [17].

To overcome this problem of Quercetin for enhancement of solubility, absorption, and biological half-life by emerging formulation strategy that is mucoadhesive nanoparticles for intranasal drug delivery. The present work was based on the enhancement of solubility and drug retention time of Quercetin by the formulation of surface modified nanoparticles with the help of single emulsion technique (solvent evaporation method).

\section{MATERIALS AND METHODS MATERIALS}

Quercetin was obtained from Merck India Pvt. Ltd. Mumbai, Eudragit ${ }^{\circledR}$ RS 100 was obtained from Chemsworth, India. Disodium dihydrogen Phosphate, Dimethyl sulfoxide, Acetonitrile were obtained from Loba Chemie Pvt. Ltd., Mumbai, India. Tetrahydrofuron, Sodium Bicarbonate, Polyvinyl alcohol, Disodium Hydrogen Phosphate were purchased from Sigma Aldrich. All other chemicals used were of analytical grade.

\section{Formulation of Nanoparticles}

The nanoparticles of Quercetin with eudragit $\AA$ RS100 were prepared using solvent evaporation/extraction technique (the single emulsion technique) [18, 19]. Normally, $25 \mathrm{mg}$ of drug with different ratios of polymer $(1: 1,1: 2,1: 3)$ were codissolved in the mixture of tetrahydrofuron $(5 \mathrm{ml})$ and acetone $(5 \mathrm{ml})$ at room temperature. The resulted solution was slowly dropped with a speed into water (25 ml) containing PVA as an external phase. During this process, the mixture was homogenized using a high speed homogenizer (IKA® Digital T18 ULTA TURRAX®, Germany), at the various agitation speeds of 10,000, 12,000 and 14,000 rpm and kept on icedwater bath. The formed oil-in-water $(\mathrm{O} / \mathrm{W})$ emulsion was gently stirred at room temperature for $24 \mathrm{~h}$ to evaporate the organic solvents. The nanosuspensions were then centrifuged at $14,000 \mathrm{rpm}, 20{ }^{\circ} \mathrm{C}$ for $20 \mathrm{~min}$. Supernatants from previous step were undergone for a further centrifugation to ensure for obtaining all the dispersed nanoparticles. The collected nanoparticles were washed 3 times with distilled water using previously described centrifugation approach and then lyophilized using lyophilizer (Labtro Freeze Dryer) [19].

Table-1: Formulation of Nanoparticles

\begin{tabular}{|l|l|l|l|l|l|l|}
\hline $\begin{array}{l}\text { Sr. } \\
\text { No }\end{array}$ & $\begin{array}{l}\text { Batch } \\
(\text { F1-F9) }\end{array}$ & $\begin{array}{l}\text { Drug (QCT) } \\
\text { mg }\end{array}$ & $\begin{array}{l}\text { Polymer } \\
(\mathbf{m g})\end{array}$ & $\begin{array}{l}\text { PVA } \\
(\mathbf{\%})\end{array}$ & $\begin{array}{l}\text { Speed of homogenizer } \\
(\mathbf{r p m})\end{array}$ & $\begin{array}{l}\text { Addition of solution } \\
(\mathbf{m l} / \mathbf{m i n})\end{array}$ \\
\hline 1 & F1 & 25 & 75 & 0.5 & 14000 & 1 \\
\hline 2 & F2 & 25 & 25 & 1 & 12000 & 1 \\
\hline 3 & F3 & 25 & 75 & 1 & 10000 & 1.5 \\
\hline 4 & F4 & 25 & 25 & 1.5 & 14000 & 1.5 \\
\hline 5 & F5 & 25 & 25 & 0.5 & 10000 & 0.5 \\
\hline 6 & F6 & 25 & 50 & 1.5 & 10000 & 1 \\
\hline 7 & F7 & 25 & 75 & 1.5 & 12000 & 0.5 \\
\hline 8 & F8 & 25 & 50 & 1 & 14000 & 0.5 \\
\hline 9 & F9 & 25 & 50 & 0.5 & 12000 & 1.5 \\
\hline
\end{tabular}

\section{Physicochemical Characterization}

\section{Particle Size and Zeta Potential}

The particle size and zeta potential analysis of optimized NPs formulation was carried out using dynamic light scattering method. The nanosuspension of nanoparticles was examined using a particle size analyzer. The equipment was sensitive in the range of 1 $\mathrm{nm}$ to $10 \mu \mathrm{m}$, and flexibility of positioning the sample to optimize the count rate using the associated software. A nano particle analyzer equipped with dynamic light scattering was used to measure zeta potential of the QCT-NPs formulation. Cell temperature was $25^{\circ} \mathrm{C}$ with a detection angle of $90^{\circ}$. All measurements were performed in triplicate. The measurements were conducted at room temperature within the equipment sensitivity range of -200 to $+200 \mathrm{mV}$.

\section{Scanning Electron Microscopy (SEM)}

Approximately $5 \mu \mathrm{g} / \mathrm{ml}$ nanosuspension of NPs was transferred to a cover slip, which in turn was mounted on a specimen tab. The samples were allowed to dry at room temperature. The particle size of the formulation was viewed and photographed using Scanning Electron Microscope. The particles were coated with platinum by using vacuum evaporator and thus, the coated samples were viewed and photographed in JEOL JSM-6701F Field Emission SEM.s

\section{Percent encapsulation efficiency and (\%EE) percent drug-loading (\%DL)}

An accurately weight amount of QCT NPs was added to $2 \mathrm{ml}$ acetonitrile and then carefully transferred to centrifugation tube. The QCT NPs in the form of pellets were separated from the solution by ultracentrifugation at $15,000 \mathrm{rpm}$ at $4^{\circ} \mathrm{C}$ for $40 \mathrm{~min}$. The supernatant $(1 \mathrm{ml})$ was carefully decanted and make the volume up to $10 \mathrm{ml}$ and analyzed by UV spectrophotometer (Model: V-630, JASCO International Co. Ltd., Tokyo Japan) [20]. The \%EE and $\% \mathrm{DL}$ was calculated using equation as given below [21], 


$$
\begin{aligned}
& \% \text { Entrapment efficiency }=\frac{\text { Total drug - Free drug }}{\text { Total drug }} \times 100 \\
& \% \text { Drug Loading }=\frac{\text { Total drug - Free drug }}{\text { Weight of nanoparticles }} \times 100
\end{aligned}
$$

\section{Fourier Transform Infrared Spectroscopy (FTIR)}

Pure quercetin, eudragit ${ }^{\circledR}$ RS 100 , the physical mixture of quercetin and Eudragit ${ }^{\circledR}$ RS 100 and the optimized NPs formulation were further chemically characterized and compared using FTIR spectroscopy. The samples were tested using FTIR spectrophotometer. Sample preparation involved preparing potassium bromide $(\mathrm{KBr})$ pellets containing $2 \mathrm{mg}$ air dried samples. Thin transparent discs of $\mathrm{KBr}$ were compressed on a Mini Hand Press at a pressure of 10 ton $/ \mathrm{Nm}^{2}$, individual samples discs were scanned within a wavelength ranging from 4000 to $400 \mathrm{~cm}^{-1}$, with a scan resolution of $4 \mathrm{~cm}^{-1}$ the obtained spectra were analyzed by the software associated with the instrument.

\section{Powder X-ray Diffraction Studies (P-XRD)}

The comparative crystalline nature of Pure quercetin, eudragit ${ }^{\circledR}$ RS 100 , the physical mixture of quercetin and Eudragit@ RS 100 and the optimized NPs formulation were studied using a PXRD on a x-ray diffractometer equipped with Braggs-Brentano geometry $(\theta / 2 \theta)$ optical setup. The method reported previously was used to analyze the samples.

\section{Aqueous Solubility Study of QCT, Optimized QCT NPs Physical Mixture (QCT and Eudragit RS 100)}

Solubility analysis of QCT, Physical mixture (QCT and Eudragit RS 100) and prepared QCT NPs in water was carried out by a method previously described in the literature ${ }^{[21]}$.Briefly the suspension of samples was prepared by adding an excess amount of QCT, Physical mixture (QCT and Eudragit RS 100) and prepared QCT NPs formulation in screw cap glass vials containing $10 \mathrm{ml}$ of distilled water. Prepared suspension in vials was then agitated on rotary shaker (Model: RS24 BL, REMI Laboratory Instruments, Remi House, Mumbai, India) at $37^{\circ} \mathrm{C}$ for a period of 24 hours. After agitation solution was then centrifuge at $10000 \mathrm{rpm}$ for 20 minutes and filtered via membrane filter $(0.45 \mu \mathrm{m})$. The filtrate was collected, withdrawn $1 \mathrm{ml}$ aliquots, diluted to $10 \mathrm{ml}$ with distilled water and assayed the resulting solution for the estimation of QCT at $389 \mathrm{~nm}$ using UV spectrophotometer (Model: V-630, JASCO International Co., Ltd., Tokyo, Japan).

\section{Functional Characterization \\ In-Vitro Dissolution Study}

The comparative in vitro release performance of QCT and prepared QCT NPs were carried out using dialysis method reported earlier [22, 23]. A dialysis membrane (LA393-5MT, dialysis membrane -70, average flat width $29.31 \mathrm{~mm}$, average diameter 17.5 $\mathrm{mm}$ and capacity $2.41 \mathrm{ml} / \mathrm{cm}$; HIMEDIA Laboratories, Mumbai, India), which used to prepare dialysis bags for the samples. The QCT as well as prepared nanoparticles, (all containing $2 \mathrm{mg}$ QCT) were added to the dialysis bag and tied the tied membrane bag was suspended into a beaker containing phosphate buffer saline $\mathrm{p}^{\mathrm{H}} 7.4$ with $0.1 \%$ Tween 80 . The contents of the beakers were stirred at $50 \mathrm{rpm}$ using magnetic stirrer at $37^{\circ} \mathrm{C}$. At predetermined time intervals, $5 \mathrm{ml}$ sample was withdrawn from the dissolution medium and replaced with an equivalent amount of release media. The drug concentrations from the aliquots were analyzed using UV spectrophotometer (Model: V-630, JASCO International Co. Ltd., Tokyo, Japan) at $370 \mathrm{~nm}$. The amount of drug released was calculated using a calibration curve of QCT in phosphate buffer at $\mathrm{pH}$ 7.4. The cumulative amount of the released QCT was calculated considering the replaced volume of the dissolution medium and the cumulative percentage of the released QCT was plotted versus time.

\section{In-Vitro Diffusion Study}

An in vitro drug release test of the nanoparticles was performed using Franz diffusion cells with dialysis membrane. A dialysis membrane (LA3935MT, dialysis membrane -70 , average flat width 29.31 $\mathrm{mm}$, average diameter $17.5 \mathrm{~mm}$ and capacity 2.41 $\mathrm{ml} / \mathrm{cm}$; HIMEDIA Laboratories, Mumbai, India), which used as a diffusion barrier. The donor compartment contained a pure QCT (25mg) and prepared QCT NPs (equivalent to $25 \mathrm{mg}$ of $\mathrm{QCT}$ ) while the receptor compartment was filled with $22 \mathrm{ml}$ phosphate buffer solution ( $\mathrm{pH} 6.6,22 \mathrm{ml}$ ) that was within the $\mathrm{pH}$ range in the nasal cavity. The donor chamber was placed in such a way that it just touched the diffusion medium in the receptor chamber. The temperature was maintained constant at $37 \pm 1^{\circ} \mathrm{C}$ using magnetic stirrer $(50 \mathrm{rpm})$. At predetermined time intervals, Samples $(1 \mathrm{ml})$ were periodically withdrawn from the receptor compartment, replaced with the same amount of fresh pre-warmed buffer solution, and assayed using a UV spectrophotometer (Model: V-630, JASCO International Co. Ltd., Tokyo, Japan) at $370 \mathrm{~nm}$ for QCT. After suitable dilutions and the concentrations of QCT were calculated using calibration curves [24].

\section{Ex- vivo Drug permeation study}

The permeation efficiency of pure QCT and QCT from prepared QCT NPs across biological membrane was studied using Franz diffusion cells. Briefly, freshly cut nasal mucosa was obtained from a local slaughterhouse, cleaned and rinsed with simulated nasal electrolyte solution. After rinsing the membrane 
was placed on the Franz diffusion cell apparatus with mucosal surface towards donor compartment and the serosal surface towards the receptor compartment. The receptor compartment was filled with phosphate buffer $(\mathrm{pH} 6.6,22 \mathrm{ml})$ maintained at $37^{\circ} \mathrm{C}$, with continuous stirring $(50 \mathrm{rpm})$. The pure QCT $(25 \mathrm{mg})$ and the prepared formulation i.e. QCT NPs (containing equivalent to $25 \mathrm{mg}$ QCT) was loaded onto the mucosal surface of the mucosal tissue (donor compartment). As per predetermined time intervals, samples $(1 \mathrm{ml})$ were periodically withdrawn from the receptor compartment, replaced with the same amount of fresh pre-warmed buffer solution, and assayed using a UV spectrophotometer (Model: V-630, JASCO International Co. Ltd., Tokyo, Japan) at $370 \mathrm{~nm}$ for QCT. After suitable dilutions and the concentrations of QCT were calculated using calibration curve [24, 25].

\section{In Vitro Mucoadhesion Study}

The mucoadhesion behavior of the prepared QCT NPs was evaluated on goat nasal mucosa using a method "Falling liquid film technique". Freshly, cut nasal mucosa was obtained from a local slaughterhouse, cleaned and rinsed with simulated nasal electrolyte solution. A $5 \mathrm{~cm}$ long strip of goat nasal mucosa was mounted on a polyethylene plate, placed an angle of $45^{\circ}$. Accurately weighed QCT NPs formulation (containing $25 \mathrm{mg}$ of QCT) was carefully sprinkled on the tissue. Phosphate buffer ( $\mathrm{pH} 6.6$, preheated at $37^{\circ} \mathrm{C}$ ) was peristaltically pumped over the mucosa at a rate of $5 \mathrm{ml} / \mathrm{min}$ for a period of 1 hour. After 1 hour, the mucosal perfusate containing non-adhered nanoparticles was collected, diluted suitably, and the absorbance of the resulting solution was analyzed using UV spectrophotometer (Model: V-630, JASCO International Co. Ltd., Tokyo, Japan) at $370 \mathrm{~nm}$ for QCT [24, 25]. The mucoadhesive potential was calculated by following formula,

\section{Amount of adhered Nanoparticles

In vitro mucoadhesion $(\%)=\frac{\text { Amount of applied Nanoparticles }}{\text { X100 }}$

\section{Histopathological Study}

Histopathological studies were carried out to investigate any adverse anatomical or structural influences of the prepared QCT NPs on the nasal mucosa. Freshly cut goat nasal mucosa was obtained from local-slaughterhouse, cleaned and rinsed with Simulated Nasal Electrolyte Solution (SNES). After thorough rinsing, the tissue was cut into two equal parts. One part of nasal mucosa was treated with QCT NPs (containing $25 \mathrm{mg}$ QCT) and the other part of nasal mucosa treated with Phosphate buffer ( $\mathrm{pH}$ 6.6) and considered as control. After treatments, the tissue was stored at ambient temperature $\left(25^{\circ} \mathrm{C}\right)$ for a period of 1 hour. After 1 hour, both the QCT NPs and phosphate buffer treated (control) tissues were preserved in neutral buffered formalin solution $(10 \%$ $\mathrm{v} / \mathrm{v})$ until additional processing, The tissue was then sectioned, and stained with hematoxylin-eosin reagent. The stained images were observed with an optical microscope (Model: DM 1802) and there images were captured to detect any damages to the tissue [22, 23].

\section{RESULTS AND DISCUSSIONS \\ Particle size and zeta potential}

The particle size and zeta are valuable predictors of effective distribution and physical stability of nanoparticles. According to Gioia et al., 2015 the particle size (100-300 nm), indicating a narrow size distribution [26]. Such particle sizes and narrow size distributions were considered favorable for intranasal administration. In the present study the formulation prepared by single emulsion technique displayed particle size at about $297.8 \pm 0.67 \mathrm{~nm}$ with polydispersity index (PDI) i.e. $0.41 \pm 0.09$ for optimized QCT NPs (batch F8) is appropriate for nasal drug delivery. The low PDI indicates narrow range of particle size distribution.

Zeta potential $(\zeta)$ an important tool used for determination of stability and surface charges on the particles. The zeta potential value in the range of -30 $\mathrm{mV}$ to $+30 \mathrm{mV}$ indicates ideal physical stability of the nanocarriers. The zeta potential value for optimized QCT NPs (F8 batch) $-11.0 \pm 0.34 \mathrm{mV}$ which is found within the desired range as above mentioned. Therefore, the obtained mean particle size, lower PDI and suitable zeta potential value demonstrates the suitability of optimized QCT NPs formulation for intranasal administration accompanied with an excellent physical stability.

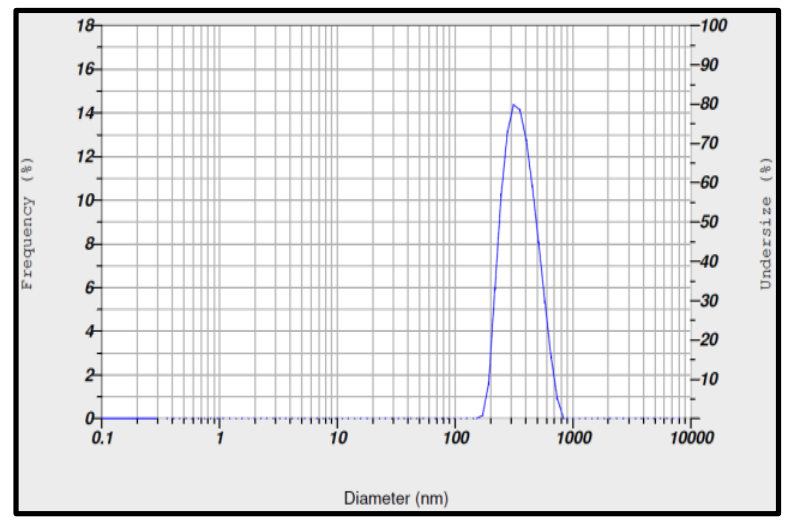

Fig-1: Particle size of optimized QCT NPs 


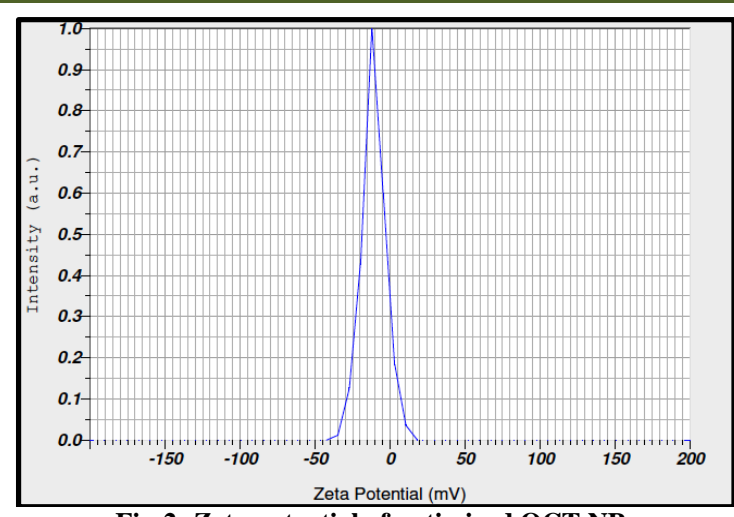

Fig-2: Zeta potential of optimized QCT NPs

\section{Scanning electron microscopy (SEM)}

Pure QCT, as shown in Figure-3, formed cuboidal particles with a wide range from several to dozens of micron. Eudragit ${ }^{\circledR}$ RS 100 as shown in Figure-4, appeared as amorphous particles as they are spherical in shape. Surface morphology of QCT NPs as examined by scanning electron microscopy was illustrated in Figure-5. The QCT NPs was made up of Quercetin and Eudragit ${ }^{\circ}$ RS 100 appeared as amorphous particles. When at $7000 \mathrm{X}$ magnifications, it could be seen that the presence of spherical shape of QCT NPs with a relative smooth surface for the resultant nanoparticle.

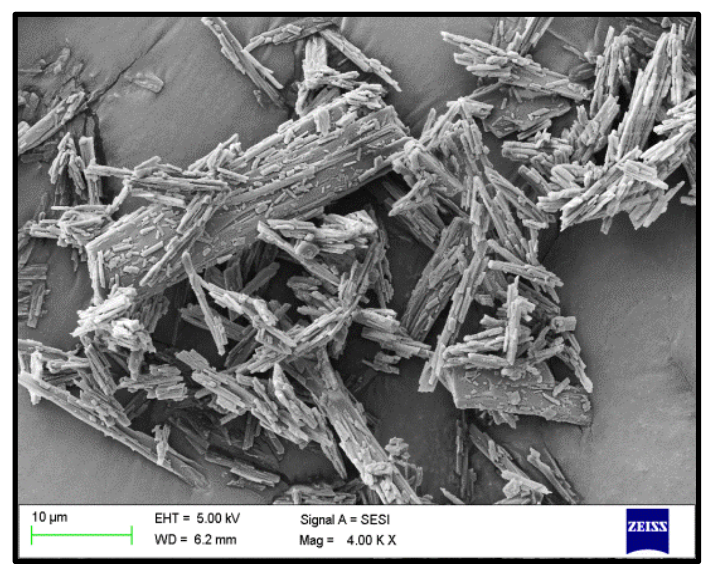

Fig-3: The scanning electron microscope images of Quercetin

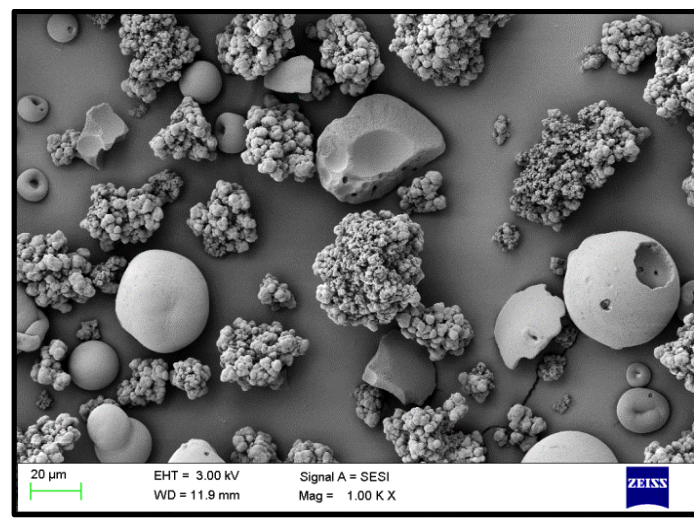

Fig-4: The scanning electron microscope image of Eudragit ${ }$ RS 100

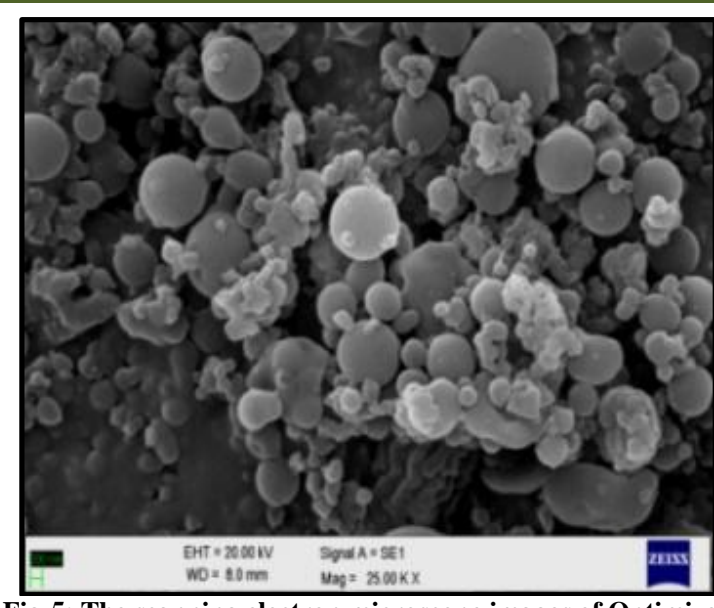

Fig-5: The scanning electron microscope images of Optimized QCT NPs

\section{Fourier transform infrared spectroscopy (FT-IR)}

The spectra obtained from the FTIR analysis of pure quercetin, Eudragit ${ }^{\circledR}$ RS 100, physical mixture of QCT and eudragit® RS 100 and QCT and QCT NPs formulation are shown in Figure 6 to 9. Pure quercetin revealed characteristic absorption bands at $3391.91 \mathrm{~cm}-$ 1 and $3257.7 \mathrm{~cm}-1$ (related to phenolic O-H stretching) (Figure 17). Other minor absorption bands observed for quercetin included the ones at $1658.7 \mathrm{~cm}-1$ and 1602.8 cm-1 (C=O stretching), $1520.8 \quad \mathrm{~cm}-1 \quad$ (aromatic stretching), $1446.2 \mathrm{~cm}-1$ and $1379.1 \mathrm{~cm}-1 \quad(\mathrm{C}-\mathrm{H}$ stretching), and $1315.8 \mathrm{~cm}-1$ and $1162.9 \mathrm{~cm}-1$ (C-O-C stretching). The Eudragit ${ }^{\circledR}$ RS 100 spectrum showed the peak at $3418.0 \mathrm{~cm}-1$ (O-H stretching), $1725.8 \mathrm{~cm}-1$ ( $\mathrm{C}=\mathrm{O}$ stretching), $11480 \mathrm{~cm}-1$ (strong C-O stretching) and $838.7 \mathrm{~cm}-1$ (strong C-Cl stretching) (Figure 18). The physical mixture of quercetin and Eudragit ${ }^{\circledR} \mathrm{RS}$ 100 exhibited absorption bands associated with both compounds i.e., at $1725.8 \mathrm{~cm}-1,1610.2 \mathrm{~cm}-1,1166.7$ $\mathrm{cm}-1,1198.8 \mathrm{~cm}-1,1013.8 \mathrm{~cm}-1,1319.5 \mathrm{~cm}-1,1561.5$ $\mathrm{cm}-1$, and $1662.67 \mathrm{~cm}-1$. The FTIR spectrum of QCT NPs formulation is shown in Figure 20. Major absorption bands were observed at $823.5 \mathrm{~cm}-1,1192.7$ $\mathrm{cm}-1,1725.8 \mathrm{~cm}-1,1166.8 \mathrm{~cm}-1,1319.5 \mathrm{~cm}-1,1298.47$ $\mathrm{cm}-1,1539.4 \mathrm{~cm}-1$, and $1666.1 \mathrm{~cm}-1$. This spectrum revealed some peaks that were unique to QCT NPs formulation, and some that were associated with either the pure components or the physical mixture of the components. The peaks associated with the pure components or the physical mixture was observed to be slightly shifted, possibly due to the weak intermolecular interactions between the two molecules during the formation of the nanoparticles. 


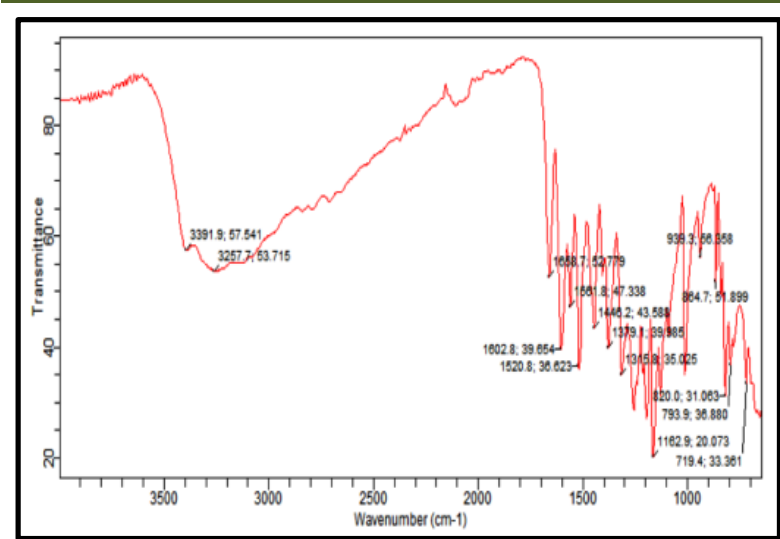

Fig-6: FTIR spectra of pure Quercetin

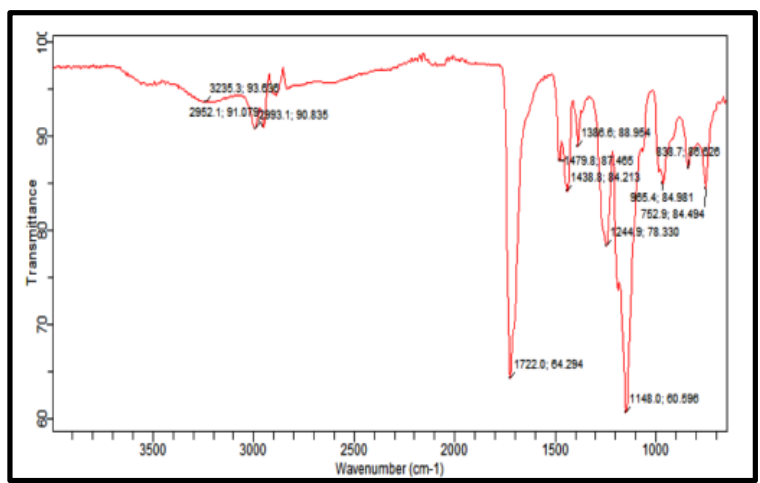

Fig-7: FTIR spectra of Eudragit RS 100

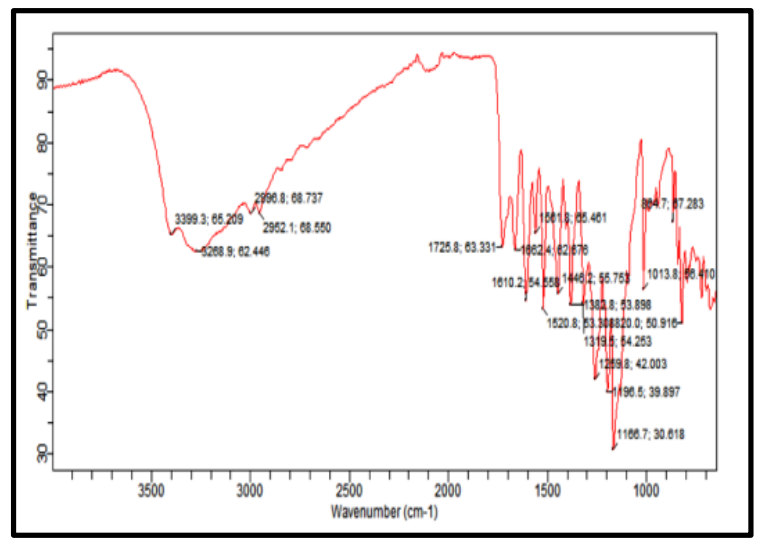

Fig-8: FTIR spectra of physical mixture (QCT and Eudragit ${ }^{\circledR}$ RS 100)

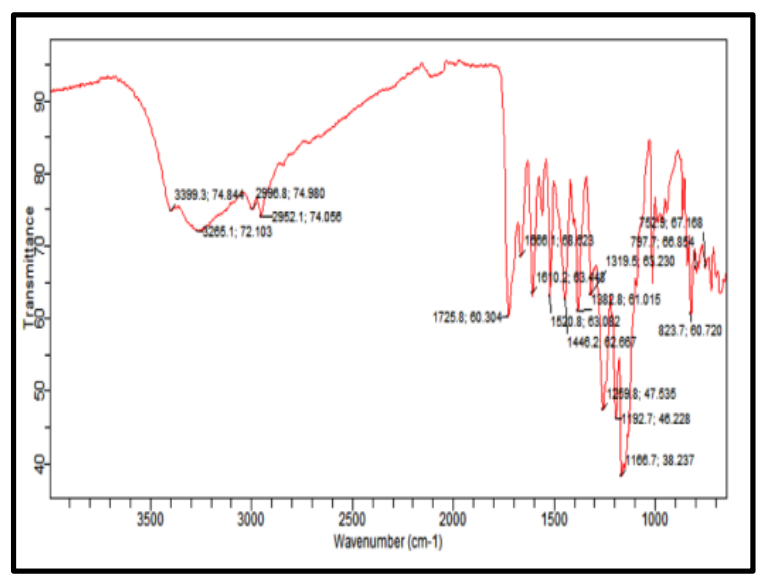

Fig-9: FTIR spectra of QCT NPs

\section{X-Ray Powder Diffraction (X-RPD)}

The comparative structural characteristics of pure quercetin, eudragit ${ }^{\circledR}$ RS 100 , physical mixture of QCT and eudragit ${ }^{\circledR}$ RS 100, and QCT NPs, as determined by PXRD analysis are shown in the form of $\mathrm{X}$-ray diffractograms in the Figure 10 to 13 . Figure 10 shows the diffractogram of pure quercetin. Quercetin exhibited five sharp peaks of high intensity at $11^{\circ}$, $12.5^{\circ}, 16^{\circ}, 24^{\circ}$, and $27^{\circ} 2 \theta$, attributable to the crystalline nature of quercetin. The diffraction pattern of eudragit ${ }^{\circledR} \mathrm{RS} 100$, revealed one peak $13.97^{\circ} 2 \theta$, indicating the partial-amorphous nature of the compound. The physical mixture of quercetin and eudragit ${ }^{\circledR}$ RS 100 showed a blend of peaks with varying intensities at $10.89^{\circ}, 12.52^{\circ}, 15.95^{\circ}, 24.09^{\circ}$ and $27.50^{\circ}$ and $2 \theta$. The low intensity peaks were similar to those observed with pure quercetin; whereas, the single broad peak can be assigned to the eudragit ${ }^{\circledR}$ RS 100 molecule. The decreased intensity of quercetin peaks in the physical mixture has been reported to be caused by the lower amount of quercetin in mixture, interference by eudragit ${ }^{\circledR}$ RS 100 molecule. Finally, the diffractogram of QCT NPs exhibited two peak at $2 \theta=$ $12.51,27.53^{\circ}$. Moreover, it is possible that the diffraction peaks observed are due to any crystalline components remaining in the prepared complex. It is assumed that, in the prepared QCT NPs, quercetin is molecularly dispersed within the polymeric matrix resulting in a change in the crystalline nature of quercetin. Not-surprisingly, the intense crystalline peaks of quercetin is thus expected to be suppressed.

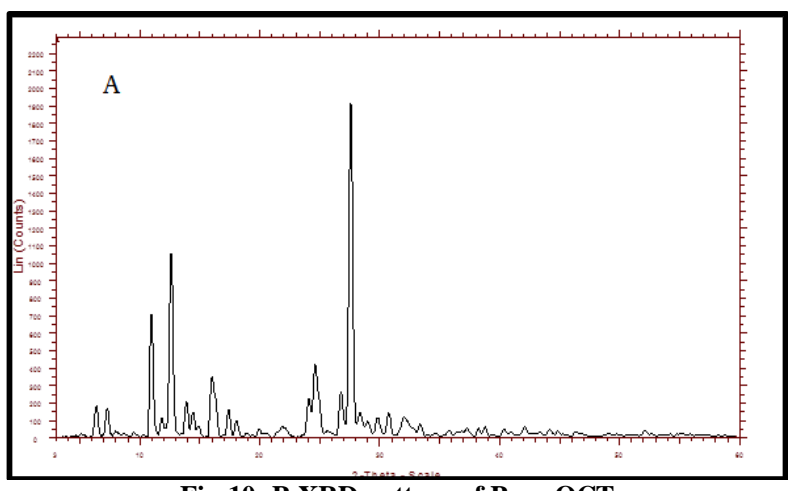

Fig-10: P-XRD pattern of Pure QCT

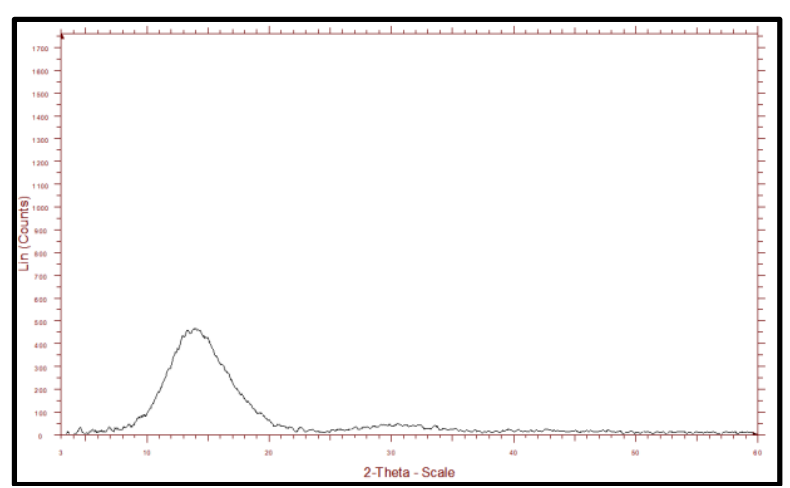

Fig-11: P-XRD pattern of Eudragit RS 100 


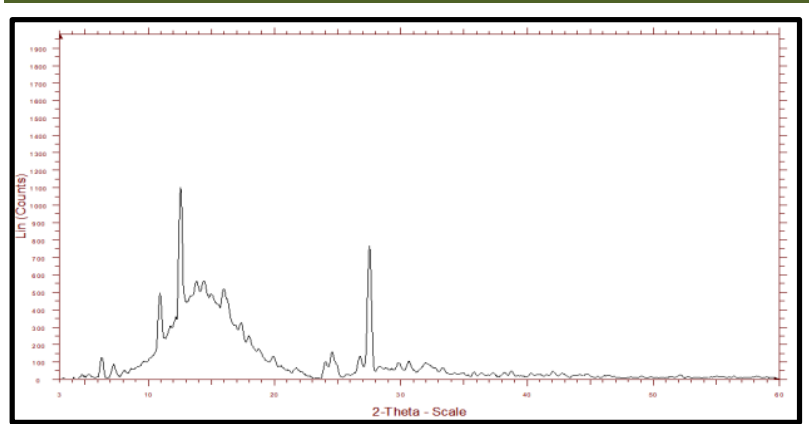

Fig-12: P-XRD pattern of PM of QCT and Eudragit ${ }^{\circledR}$ RS 100

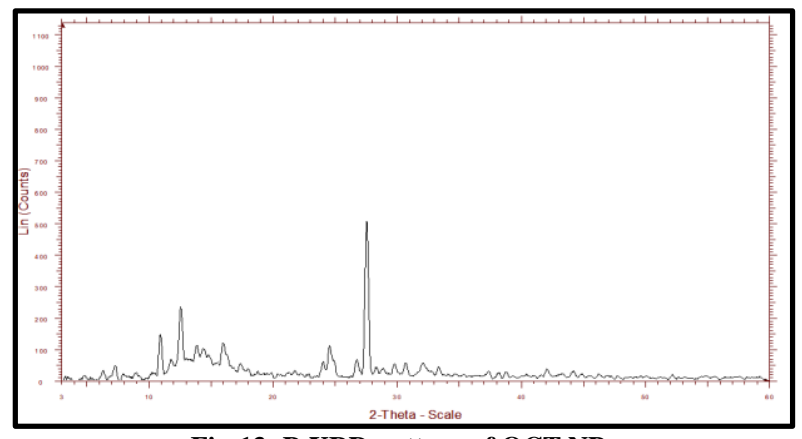

Fig-13: P-XRD pattern of QCT NPs
Solubility study of QCT, optimized nanoparticles and physical mixture (QCT and eudragit $\left.{ }^{\circ} \mathrm{RS} 100\right)$

The results of the measured solubility of the pure QCT, PM and QCT NPs in water are shown in Table-2. Pure QCT was observed to have poor aqueous solubility $(2.55 \pm 0.76 \mu \mathrm{g} / \mathrm{ml})$, and the physical mixture (PM) revealed significant increase in the aqueous solubility compared to that of pure QCT $(2.55 \pm 0.76$ $\mu \mathrm{g} / \mathrm{ml}$ ). Though the optimized (F8) QCT NPs formulation (based on highest entrapment efficiency and drug loading) exhibited a significant elevated aqueous solubility $(61.27 \pm 0.89 \mu \mathrm{g} / \mathrm{ml})$ in the aqueous solubility compared to that of pure QCT $(2.55 \pm 0.76$ $\mu \mathrm{g} / \mathrm{ml})$ and physical mixture. Partial amorphization (reduced molecular crystallinity) of QCT, and the overall nature of surface modified nanoparticles showed increase in aqueous solubility. The solubility of pure QCT, PM and QCT NPs performed in distilled water at $25^{\circ} \mathrm{C}$. The results indicated that the solubility of QCT NPs in distilled water was improved ( 24 fold) in comparison to pure QCT. And the solubility of physical mixture in distilled water was improved $(\sim 11.26)$ in comparison to pure QCT

Table-2: Solubility study of QCT, optimized Nanoparticles and physical mixture (QCT and Eudragit ${ }^{\circledR}$ RS 100)

\begin{tabular}{|l|l|l|}
\hline Sr. No & Samples & $\begin{array}{l}\text { Solubility in distilled water } \\
\boldsymbol{\mu g} / \mathbf{m l} \pm \text { SD }\end{array}$ \\
\hline 1 & Pure Quercetin & $2.55 \pm 0.76 \mu \mathrm{g} / \mathrm{ml}$ \\
\hline 2 & PM of QCT and Eudragit@ RS 100 & $28.73 \pm 0.45 \mu \mathrm{g} / \mathrm{ml}$ \\
\hline 3 & QCT NPs & $61.27 \pm 0.89 \mu \mathrm{g} / \mathrm{ml}$ \\
\hline
\end{tabular}

Estimation of entrapment efficiency and drug loading

Results of QCT in prepared QCT NPs formulations are shown in Table-3. The entrapment efficiency and drug loading of QCT in all prepared QCT NPs formulations i.e. F1 to F9, was found to be in the range 65.32 to $97.26 \% \mathrm{w} / \mathrm{w}$ and 11.70 to $42.13 \%$ w/w respectively. However, the F8 batch (nanoparticles prepared with the agitation speed of $14,000 \mathrm{rpm}, 1: 2$ drug: polymer ratio, $1 \% \mathrm{w} / \mathrm{v}$ PVA concentration, and the $0.5 \mathrm{ml} / \mathrm{min}$ solution addition speed) exhibited highest entrapment efficiency $(97.39 \pm 0.71 \% \mathrm{w} / \mathrm{w})$ and drug loading $(42.13 \pm 1.28 \% \mathrm{w} / \mathrm{w})$, and thus selected as optimized formulations were preferred for further assessments due to their highest entrapment efficiency and drug loading.

Table-3: Entrapment efficiency and Drug loading of bath F1 to F9

\begin{tabular}{|l|l|l|l|}
\hline Sr. No & Formulation code & Entrapment Efficiency $(\% \mathbf{w} / \mathbf{w})$ & Drug Loading $(\% \mathbf{w} / \mathbf{w})$ \\
\hline 1 & F1 & $95.20 \pm 0.89$ & $35.39 \pm 1.12$ \\
\hline 2 & F2 & $89.16 \pm 1.23$ & $22.28 \pm 0.94$ \\
\hline 3 & F3 & $78.30 \pm 2.39$ & $19.46 \pm 1.53$ \\
\hline 4 & F4 & $90.16 \pm 1.68$ & $34.99 \pm 0.87$ \\
\hline 5 & F5 & $65.39 \pm 0.45$ & $11.70 \pm 2.36$ \\
\hline 6 & F6 & $86.52 \pm 1.79$ & $24.21 \pm 1.09$ \\
\hline 7 & F7 & $81.23 \pm 1.54$ & $22.65 \pm 0.59$ \\
\hline 8 & F8 & $97.26 \pm 0.71$ & $42.13 \pm 1.28$ \\
\hline 9 & F9 & $86.92 \pm 0.67$ & $24.30 \pm 0.72$ \\
\hline
\end{tabular}

\section{In vitro dissolution studies}

The result of the comparative dissolution profiles of pure QCT and prepared QCT NPs formulations i.e. batch $\mathrm{F} 1$ to $\mathrm{F} 9$ in phosphate buffer saline (7.4) are shown in figure 6.25. The release profiles of QCT appeared up to $49.53 \pm 1.25 \%$ at 12 hrs. This lower amount of release in dissolution media could be due to poor aqueous solubility of QCT. Prepared QCT NPs formulations were showed an enhanced rate and extent of dissolution. QCT NPs formulations i.e. batch F1, F2, F3, F4, F5, F6, F7 and F9 demonstrated an enhanced dissolution rate as compared to pure QCT. Amongst these formulations Batch F8 showed an excellent release rate at about 
$75.57 \pm 0.878 \%$ up to 12 hrs. Pure QCT has low aqueous solubility which is shown to improve by the Eudragit ${ }^{\circledR}$ RS 100; QCT is assumed to change from crystalline state to a partially amorphous state in the QCT NPs., which may extend the rate and extent of dissolution up to $12 \mathrm{hrs}$; and change in the structural morphology of crystalline QCT improved solubility.

Various kinetic models viz., zero order, first order, matrix, korsmeyer-peppas and Hixon crowell were explored to determine the model fit of the release profile of QCT NPs. Korsmeyer-Peppas model was found to be the best representative model for F8 batch, describing the dissolution of QCT NPs, based on the obtained value of the regression coefficient (R2=0.9997). Additionally, the release exponent value (n) was estimated to be 1.1385 thus indicating a nonFickian diffusion release. Hence QCT NPs was found to significantly enhance both the rate and extent of QCT release compared to pure QCT.
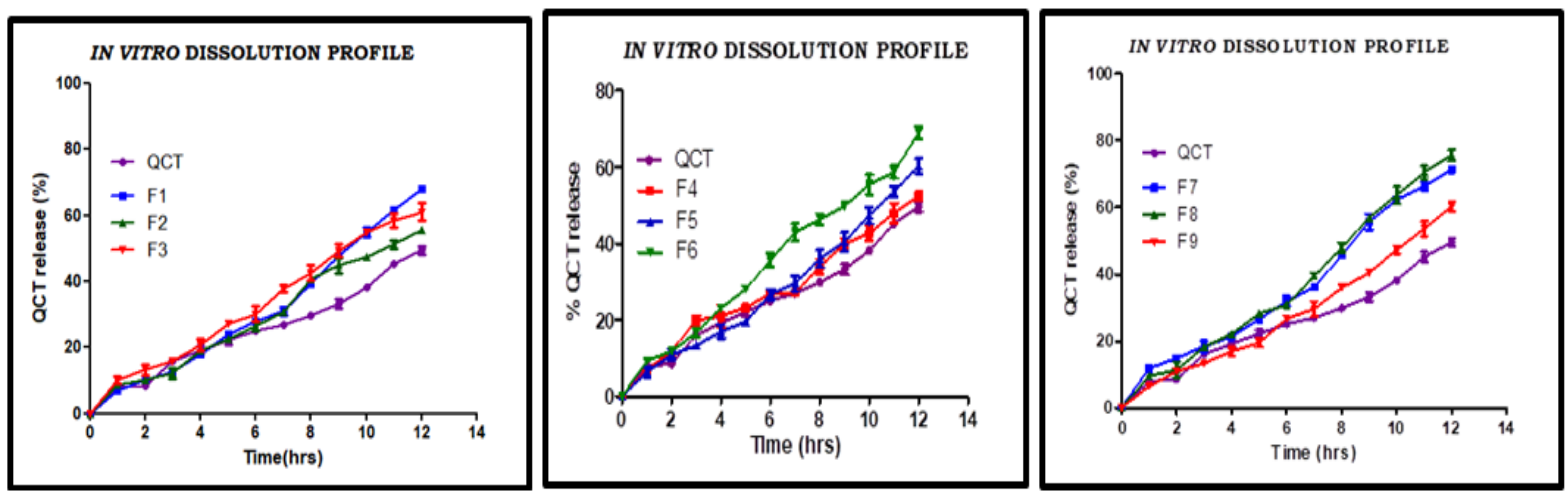

Fig-14: The in vitro dissolution profiles of QCT release from QCT suspension and QCT NPs formulation (batch F1 to F9). Values are mean \pm Std. $\operatorname{dev}(n=3)$

\section{In vitro diffusion studies}

The drug release characteristics of the formulation were studied in In vitro conditions by using semipermeable membrane in PBS pH 6.6. The release profiles of QCT appeared up to $39.49 \pm 0.8897 \%$ at 12 hrs. QCT NPs has shown release of $51.47 \pm 0.7929 \%$ at $11 \mathrm{hrs}$; and $63.56 \pm 0.3615 \%$ at 12 hrs. Thus, QCT NPs was found to significantly enhance both the rate and extent of QCT release compared to pure QCT. Various kinetic models viz., Zero order, First order, matrix, Korsmeyer-Peppas and Hixon-Crowell were explored to determine the model fit of the release profile of QCT NPs. Based on the obtained value of the regression coefficient $(\mathrm{R}=0.9573)$, Zero order kinetics model was found to be the best representative model describing the dissolution of QCT NPs. Additionally, the release exponent value (n) was estimated to be 0.8464 , thus indicating a non-Fickian diffusion release. Hence QCT NPs was found to significantly enhance both the rate and extent of QCT release compared to pure QCT.

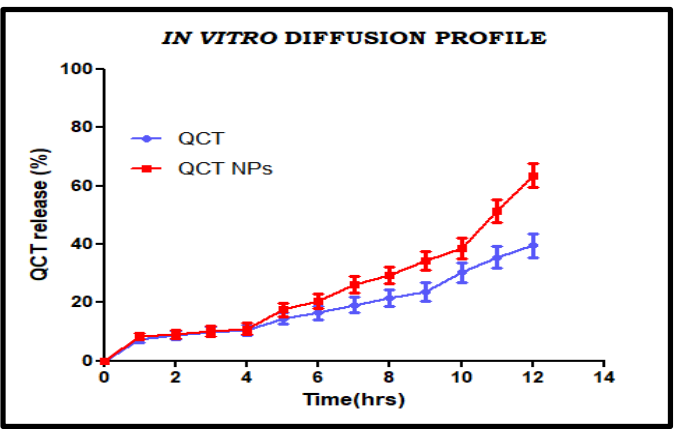

Fig-15: The in vitro drug diffusion profile of QCT release from QCT suspension and QCT NPs formulation (F8 batch).Values are mean \pm Std. $\operatorname{dev}(n=3)$

\section{In vitro mucoadhesion studies}

The in vitro mucoadhesion study was executed to ensure the adhesion of formulation to the nasal mucosa for a prolonged period of time at the site of absorption. Result suggested that the QCT NPs had satisfactory mucoadhesion properties $73.45 \pm 1.16 \%$ and could adequately adhere on the goat nasal mucosa. The mucoadhesive property of formulation depends on the presence of functional groups favoring mucoadhesion by interaction with mucin and polymers rich in aforesaid functional groups which shows higher percentage of mucoadhesion.

\section{Ex-vivo permeation studies}

The pure drug and optimized formulation was further subjected to ex vivo permeation studies using the goat nasal mucosa in PBS pH 6.6. The formulation (QCT NPs) shows $67.79 \pm 0.4796 \%$ of drug permeated in 12 hrs. Various kinetic models viz., zero order, first order, matrix, korsmeyer-peppas and Hixon crowell were explored to determine the model fit of the release profile of QCT NPs. The permeation profiles of QCT follow zero order kinetics model as it evident by correlation coefficients $(\mathrm{R}=0.9845)$. The release exponent value (n) was estimated to be 0.7955 , thus indicating a non-fickian diffusion release. Hence, QCT NPs was found to be significantly enhanced both rate and the extent of QCT permeation through goat nasal mucosa compared to pure QCT. The results of drug permeation from QCT NPs through the goat nasal mucosa confirmed that QCT was released from formulation and permeated through the goat nasal 
mucosa, hence could possibly permeate through the human skin.

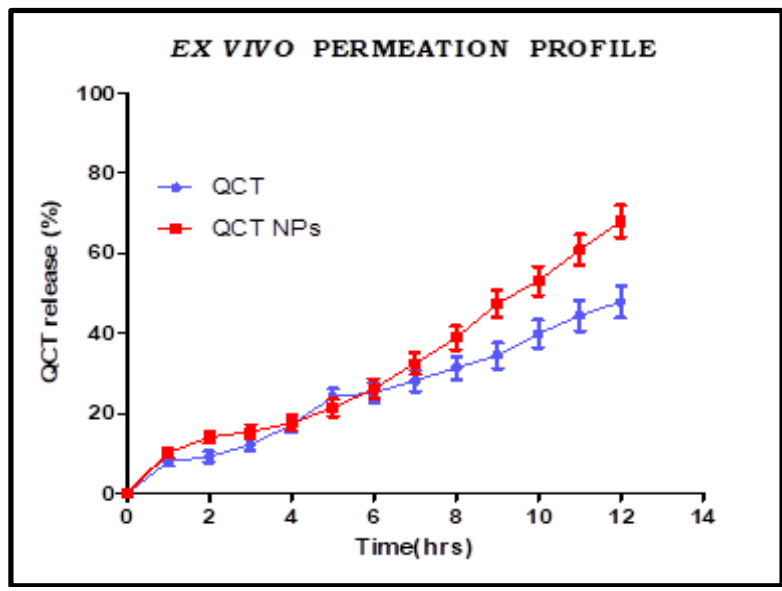

Fig-16: The ex vivo drug permeation study of QCT release from QCT suspension and QCT NPs formulation. Values are mean \pm Std. $\operatorname{dev}(\mathbf{n}=3)$

\section{Histopathological studies of nasal mucosa}

It is necessary to examine histological changes in nasal mucosa caused by formulations, if it is to be considered for practical use. Histological studies showed control mucosa that is normal nasal mucosa stained with hematoxylin-eosin (Figure-17A) and the effect of formulation on goat nasal mucosa, 1 hour after applying the QCT NPS (Figure-17B). No change in mucosal structure was seen when treated with formulation as compared to the control. Ciliated respiratory epithelium and normal goblet cell were observed. This confirms that formulation does not cause any deleterious response and adverse effect on nasal mucosa. No severe signs of damage such as appearance of epithelial necrosis or sloughing of epithelial cells were detected on the integrity of nasal mucosa. The epithelium layer was intact and there were no alterations in basal membrane and superficial part of sub-mucosa as compared with PBS treated mucosa. Thus, in-situ gel formulations seem to be safe with respect to nasal administration.

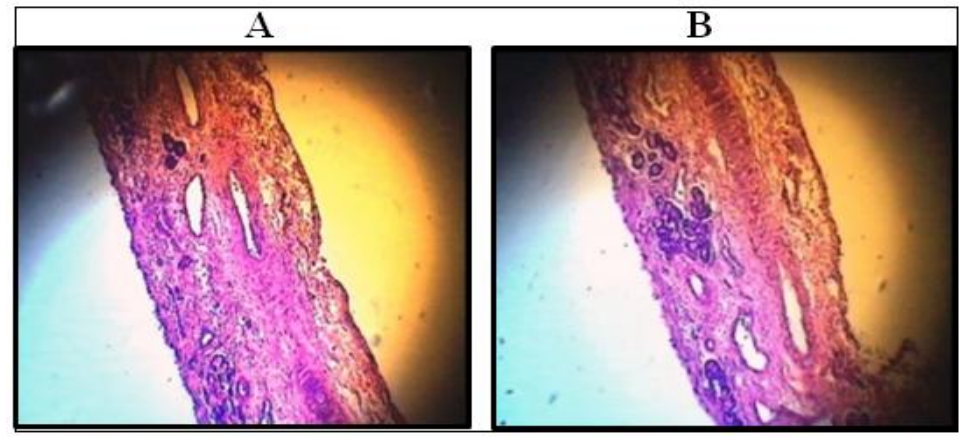

Fig-17: Light photomicrograph of the nasal mucosa [Untreated mucosa (A) and QCT NPs treated mucosa (B)]

\section{CONCLUSION}

The particle size, zeta potential and SEM showed the Nano form and amorphous nature of formulation. The physical-chemical characterization including FTIR and P-XRD of the prepared formulation supported the formation of nanoparticles, and indicated involvement of weak intermolecular interactions between quercetin and eudragit ${ }^{\circledR}$ RS 100. Optimized QCT NPs formulation showed extended release of QCT than pure QCT suspension. The in vitro diffusion study of QCT NPs formulation showed extended release of QCT than pure QCT until $12 \mathrm{hrs}$. The in vitro mucoadhesion study of NPs formulation showed satisfactory mucoadhesive property by adhering the formulation to goat nasal mucosa. The ex-vivo skin drug permeation study of optimized QCT NPs formulation (F8 batch) showed extended skin permeation of QCT than pure QCT until $12 \mathrm{hrs}$. The histopathology study of NPs formulation showed no structural change in epithelium i.e., no damage of mucosa found. Therefore, it can be concluded that the above NPs formulation has the potential for nose to brain drug delivery improved permeation profile for longer period of time and thereby increasing the patience compliance.

\section{ACKNOWLEDGEMENTS}

The authors would like to thank Principal, S. K. B. College of pharmacy Kamptee, India, for providing necessary facilities to carry out this research study.

\section{REFERENCES}

1. Lochhead $\mathrm{J}$, Thorne R. Intranasal delivery of biologics to the central nervous system. Advanced Drug Delivery, 2012; 64(7):614-628.

2. Mistry A, Stolnik S, Illum L. Nanoparticles for direct nose-to-brain delivery of drugs. International Journal Pharm, 2009; 379(1):146157.

3. Kulkarni A, Vanjari Y, Sancheti K, Belgamwar V, Surana S, Pardeshi C. Nanotechnology-mediated nose to brain drug delivery for Parkinson's disease: a mini review. Journal of Drug Targeting, 2015;23(9):775-788.

4. Djupesland G. Nasal drug delivery devices: characteristics and performance in a clinical perspective-a review. Drug Delivery Translational Research. 2011;3(1):42-62.

5. Jafarieh O, Md S, Ali M, Baboota S, Sahni JK, Kumari B, Bhatnagar A, Ali J. Design, 
characterization, and evaluation of intranasal delivery of ropinirole-loaded mucoadhesive nanoparticles for brain targeting. Drug development and industrial pharmacy. 2015 Oct 3;41(10):1674-81.

6. Dillen K, Vandervoort J, Van den M, Ludwig A. Evaluation of ciprofloxacin-loaded eudragit RS 100 or RL 100/PLGA nanoparticles. International Journal Pharm. 2006;314: 72-82.

7. Jana U, Mohanty A, Mohanta G. Preparation and characterization of nebivolol nanoparticles using Eudragit ${ }^{\circledR}$ RS 100. Colloids and Surfaces B: Biointerfaces, 2014; 113: 269-275.

8. Jana U, Mohanty A, Pal S, Manna P, Mohanta G. Preparation and in vitro characterization of felodipine loaded eudragit ${ }^{\circledR}$ RS100 nanoparticles. International Journal of Pharmacy and Pharmaceutical Sciences, 2014: 6(4): 564-567.

9. Russo, M., Spagnuolo, C., Tedesco, I., Bilotto, S, Russo G. The flavonoid quercetin in disease prevention and therapy: Facts and fancies. Biochemical Pharmacology 2012; 83(1): 6-15.

10. Firdous, A, Sharmila, G, Balakrishnan S, RajaSingh P, Suganya S, Srinivasan N. Quercetin, a natural dietary flavonoid, acts as a chemo preventive agent against prostate cancer in an in vivo model by inhibiting the EGFR signaling pathway. Food \& Function, 2014; 5(10): 26322645.

11. Chis I, Baltaru, D, Maier M, Clichici, S. Effects of quercetin and chronic (training) exercise on oxidative stress status in animals with streptozotocin-induced diabetes. Bulletin of University of Agricultural Sciences and Veterinary Medicine Cluj-Napoca. Veterinary Medicine, (2013); 70(1): 31-39.

12. Caridi D, Trenerry V, Rochfort S, Duong S, Laugher D, Jones R. Profiling and quantifying quercetin glucosides in onion (Allium cepa L.) varieties using capillary zone electrophoresis and high performance liquid chromatography. Food Chemistry, 2007; 105(2): 691-699.

13. Price R, Casuscelli F, Colquhoun J and Rhodes C: Composition and content of flavonol glycosides in broccoli florets (Brassica olearacea) and their fate during cooking. Journal of the Science of Food and Agriculture, 1998; 77(4): 468-472.

14. Lauro M, Maggi L, Conte U, De Simone F, Aquino R. Rutin and quercetin gastro-resistant microparticles obtained by spray-drying technique. Journal of Drug Delivery Science and Technology, 2005; 15(5): 363-369.

15. Srinivasn, K, King J, Howard L, Monrad, K. Solubility and solution thermodynamic properties of quercetin and quercetin dihydrate in subcritical water. Journal of Food Engineering, 2010; 100(2): 208-218.
16. Gilley A, Arca C, Nichols L. Novel cellulosebased amorphous solid dispersions enhance quercetin solution concentrations in vitro. Carbohydrate Polymers, 2017; 157: 86-93.

17. Cai X, fang Z, Dou J, Zhai G. Bioavailability of quercetin: problems and promises. Current medicinal chemistry, 2013; 20(20): 2572-2582.

18. Naik B, Lokhande B, Mishra S, Kulkarni D. Development of sustained release micro/nanoparticles using different solvent emulsification technique: A review. International Journal Pharm Bio Sci, 2012; 3:573-590.

19. Adibkia K, Javadzadeh Y, Dastmalchi S, Mohammadi G. Naproxen-Eudragit@ RS 100 nanoparticles preparation and its characterization. Biointerfaces, 2011;83:155.

20. Shadab M, Bhattmisra K, Zeeshan F, Shahzad N, Ali M. Nano-carrier enabled drug delivery systems for nose to brain targeting for the treatment of neurodegenerative disorders. Journal of Drug Delivery Science and Technology, 2017;17:1-61.

21. Patra A, Satpathy S, Shenoy K, Bush A, Kazi M, Hussain D. Formulation and evaluation of mixed polymeric micells of quercetin for the treatment of breast, ovarian and multidrug resistant cancers. International Journal of Pharmacy and Pharmaceutical Sciences, 2018;13:2869-2881.

22. Shijie $\mathrm{Xu}$, Fangbo C, Huang D, Zhang D, Yiming C. PD-L1 monoclonal antibody-conjugated nanoparticles enhance drug delivery level and chemotherapy efficacy in gastric cancer cells. International Journal of Nanomedicine, 2019; 14:17-32.

23. Jafarieh O, Shadab M, Ali M, Baboota S, Sahni J, Kumari B. Design, characterization, and evaluation of intranasal delivery of ropiniroleloaded mucoadhesive nanoparticles for brain targeting. Drug Development and Industrial Pharmacy, Informa Healthcare, 2014; 1-8.

24. Belgamwar V, Patel H, Joshi A, Agrawal A, Surana S. Design and development of nasal mucoadhesive microspheres containing tramadol $\mathrm{HCl}$ for $\mathrm{CNS}$ targeting. Drug Delivery, Informa Healthcare, 2011;18(5):353-360.

25. Pardeshi C, Rajput P, Belgamwar V, Tekade A. Formulation, optimization and evaluation of spraydried mucoadhesive microspheres as intranasal carriers for Valsartan. Journal of Microencapsulation, Informa Healthcare, 2012; 29(2):103-114.

26. Gioia S, Trapani T, Mandracchia D, Giglio E, Cometa S. Intranasal delivery of dopamine to the striatum using glycol chitosan/sulfobutylether-bcyclodextrin based nanoparticles. European Journal of Pharmaceutics and Biopharmaceutics, 2015; 94:180-193. 